\title{
A note on averaging correlations
}

\author{
RALPH A. ALEXANDER \\ University of Akron, Akron, Ohio
}

\begin{abstract}
Researchers are often faced with attempting to estimate a correlation based on the aggregation of sample $r$ s either from several samples or from repeated measures within a single sample. The usual approach is either to average the observed correlations or to average the Fisher's $z$ transformed $r \mathrm{~s}$ and to back-transform the average $z$ value. This note describes a minimum-variance unbiased estimator for the situation that is superior to either approach and is also simple to compute.
\end{abstract}

Although it is obviously desirable to base an estimate of the correlation between two variables on large sample sizes, this is not always possible. Often, though, it is possible to obtain correlation estimates from several samples or from repeated measurement on the same sample. In such instances, an aggregation of those sample values is useful.

Two commonly used methods for averaging such values were recently investigated in some detail by Dunlap, Jones, and Bittner (1983) and by Silver and Dunlap (1987). The first method is simply the average of observed sample $r s$ or, in the case of unequal sample sizes (Alexander, Scozzaro, \& Borodkin, 1989),

$$
\bar{r}=\frac{\Sigma\left(n_{i}-1\right) r_{i}}{\Sigma n_{i}-k},
$$

where $k=$ the number of individual sample $r$ s being aggregated and $n_{i}$ is the $n$ size for the $i$ th sample $r$.

The second method is to transform each of the $r$ s to a Fisher's $z$,

$$
z_{i}=\frac{1}{2} \log _{e}\left(\frac{1+r_{i}}{1-r_{i}}\right)=\tanh ^{-1}\left(r_{i}\right),
$$

and to average these $z$ values, which for unequal $n \mathrm{~s}$ becomes,

$$
\bar{z}=\frac{\Sigma\left(n_{i}-3\right) z_{i}}{\Sigma n_{i}-3 k} .
$$

Finally, $\bar{z}$ is back-transformed to $\bar{r}$ by

$$
\bar{r}^{\prime}=\frac{e^{2 \bar{z}}-1}{e^{2 \bar{z}}+1}=\tanh (\bar{z}) .
$$

Silver and Dunlap (1987) empirically investigated the efficacy of both methods and reported the expected results. An aggregate based on $z$-transformed values $\left(\bar{r}^{\prime}\right)$ was less biased than $\bar{r}$. The bias in $\bar{r}^{\prime}$ is positive ( $\bar{r}^{\prime}$ typically overestimates $\varrho$ ), whereas that in $\bar{r}$ is negative, and $\bar{r}^{\prime}$ has a larger standard error than $\bar{r}$.

Requests for reprints should be addressed to Ralph A. Alexander, Department of Psychology, University of Akron, Akron, OH 44325.
Hotelling (1953) proposed an improvement on Fisher's $z$ transformation. Alexander, Hanges, and Alliger (1985) found that Fisher's $z$ performed as well as Hotelling's improved transformation.

There is an alternative estimator that is superior to either $\bar{r}$ or $\bar{r}^{\prime}$. Olkin and Pratt (1958) proposed an approximately unbiased minimum-variance estimator of $\varrho$, which, on aggregation over sample values is

$$
\bar{r}^{*}=\frac{\Sigma\left(n_{i}-1\right)}{\Sigma n_{i}-k}\left\{r_{i}+\left[\frac{r_{i}\left(1-r_{i}^{2}\right)}{2\left(n_{i}-3\right)}\right]\right\} .
$$

This estimator is less biased than either $\bar{r}$ or $\bar{r}^{\prime}$ (Viana, 1982), and the direction of its bias is to underestimate 0 . The variance of $\bar{r}^{*}$ is approximately $\left(1-\varrho^{2}\right)^{2} /\left(\sum n_{i}-k\right)$, which is also the approximate variance in $\bar{r}$, whereas the variance in $\bar{r}^{\prime}$ is slightly larger (Silver \& Dunlap, 1987). Thus, the standard error or $\bar{r}^{*}$ is on the order of that of $\bar{r}$ and is smaller than $\bar{r}^{\prime}$.

Although Equation 5 provides an approximately unbiased minimum-variance estimate of $\varrho$ and is therefore preferred for averaging correlations either across samples or over repeated measures within sample, that is the only purpose it should be used for. In particular, it should not be used for other purposes in validity generalization (meta-analysis) studies since its sampling distribution suffers from the same deficiencies as does $r$ itself. Thus, using $\bar{r}^{*}$ or its single-sample analogue to test for homgeneity of effect sizes or to estimate variance in $r$ s attributable to artifacts will be just as biased as using the $r s$ themselves (Alexander et al., 1989).

\section{REFERENCES}

Alexander, R. A., Hanges, P. J., \& Alliger, G. M. (1985). An empirical examination of two transformations of sample correlations. Educational \& Psychological Measurement, 45, 797-801.

Alexander, R. A., Scozzaro, M. J., \& Borodkin, L. J. (1989). A statistical and empirical examination of the $\chi^{2}$-test for homogeneity of correlations in meta-analysis. Psychological Bulletin, 106, 329-331.

Dunlap, W. P., Jones, M. B., \& BitTner, A. C. (1983). Average correlations vs. correlated averages. Bulletin of the Psychonomic Society, 21, 213-216.

Hotelling, H. (1953). New light on the correlation coefficient and its transformations. Journal of the Royal Statistical Society, 15, 193-225. 
OlkIN, I., \& PRATT, J. W. (1958). Unbiased estimation of certain correlation coefficients. Annals of Mathematical Statistics, 29, 201-211. Silver, N. C., \& DunlaP, W. P. (1987). Averaging correlation coefficients: Should Fisher's $z$ transformation be used? Journal of Applied Psychology, 72, 146-148.
Viana, M. A. G. (1982). Combined estimators for the correlation coefficient. Communications in Statistics: Theory \& Methods, 11, 1843-1904.

(Manuscript received December 16, 1989.) 